\title{
Article \\ A New Technique for Computed-Tomography Urethrography in Males: The Clamp Method
}

\author{
Juan de Dios Berná-Mestre ${ }^{1,2}$, Florentina Guzmán-Aroca ${ }^{1,2}$, Alejandro Puerta-Sales ${ }^{1}$, Antonio Navarro-Baño ${ }^{1}$, \\ Guillermo Carbonell-López del Castillo ${ }^{1,2}$, Juan de Dios Berná-Serna ${ }^{1,2}$ and Miguel Alcaraz ${ }^{2, *}$ \\ 1 Department of Radiology, Virgen de la Arrixaca University Hospital, Ctra. Madrid-Cartagena, \\ 30120 Murcia, Spain; juandeberna@um.es (J.d.D.B.-M.); florentina.guzman@um.es (F.G.-A.); \\ alejandro.puerta@carm.es (A.P.-S.); antonio.navarro20@carm.es (A.N.-B.); guilleclc@gmail.com (G.C.-L.d.C.); \\ jdberna@um.es (J.d.D.B.-S.) \\ 2 Radiology and Physical Medicine Department, School of Medicine, University of Murcia, 30100 Murcia, Spain \\ * Correspondence: mab@um.es; Tel.: +34-868-883-601
}

check for updates

Citation: Berná-Mestre, J.d.D.; Guzmán-Aroca, F.; Puerta-Sales, A.; Navarro-Baño, A.; Castillo, G.C.-L.d.; Berná-Serna, J.d.D.; Alcaraz, M. A New Technique for Computed-Tomography Urethrography in Males: The Clamp Method. Appl. Sci. 2021, 11, 1006. https://doi.org/10.3390/app11031006

Academic Editor: Bernhard Baumann Received: 28 December 2020

Accepted: 20 January 2021

Published: 22 January 2021

Publisher's Note: MDPI stays neutral with regard to jurisdictional claims in published maps and institutional affiliations.

Copyright: (c) 2021 by the authors. Licensee MDPI, Basel, Switzerland. This article is an open access article distributed under the terms and conditions of the Creative Commons Attribution (CC BY) license (https:/ / creativecommons.org/licenses/by/ $4.0 /)$.
Featured Application: The clamp method for CT-Urethrography is easy and convenient, it proves more accurate than urethrography for measuring anterior urethral stenosis and it has better diagnostic efficacy in cases with periurethral fistula, previous urethroplasty, urethral stent or urethral lithiasis.

Abstract: The aim of the present study is to describe and evaluate a new technique for performing Computed-Tomography Retrograde Urethrography (CT-RUG). Males with urethral anomalies detected by retrograde urethrography (RUG) and/or retrograde sonourethrography (RSUG) underwent CT-RUG using the clamp method and three radiologists evaluated the anomalies in each technique separately and blindly. CT-RUG was done successfully in all the cases $(n=22)$, with means of 6 min duration and $95 \mathrm{~mL}$ of contrast; no pain was reported by $81 \%$ of the patients (VAS: 0 ) and very mild pain by the rest (VAS: 0.5-1.2). CT-RUG showed better diagnostic efficacy in cases of periurethral fistula $(n=8)$, urethral stent $(n=3)$, previous urethroplasty and urethral lithiasis $(n=2)$, a similar accuracy to RSUG for measuring the length of anterior urethral strictures $(n=9)$ and greater accuracy than RUG ( $p=0.008)$. Six cases received 2 CT sweeps, with an effective dose of $4.96 \mathrm{mSv}$, and the remaining 16 had 1 sweep and received $3.456 \mathrm{mSv}$. To the best of our knowledge, this is the first study to describe the clamp method for CT-RUG, a method that is effective and comfortable for both the patient and the operator (retrograde infusion of contrast).

Keywords: urethra; stenosis; computed tomography; fistula; stent; urethrography

\section{Introduction}

The most widely used technique for an imaging diagnosis of male urethral anomalies is still urethrography, which is divided into retrograde urethrography (RUG), to evaluate the anterior urethra, and voiding cystourethrography (VCUG), to evaluate the bladder and posterior urethra [1-3]. Detection of an anterior urethral stricture on RUG is an indication for retrograde sonourethrography (RSUG), as it gives a more accurate measurement and visualizes the surrounding spongiofibrosis, which is fundamental for planning treatment [1-3]. Computed-tomography urethrography (CT-UG) is rarely used and its usefulness is limited to the evaluation of traumatic urethral injuries and periurethral fistulas [3-5].

To achieve adequate distension of the anterior urethra (penile and bulbar) as far as the narrow external sphincter (proximal anatomical stop) a distal stop is needed to avoid contrast running out of the meatus. The conventional technique uses a Foley catheter, but the drawbacks are that the patient notes discomfort on distension of the balloon in the fossa navicularis (occasionally urethrorrhagia) and it is not useful in cases with urethromeatal anomalies (meatal stenosis, hypospadias, meatotomy, etc.) [2,6]. The clamp method is the 
newest technique for performing both urethrography and sonourethrography; it uses a device (a ring to apply external compression at the balanoprepucial sulcus) coupled to a fine-caliber (6Fr) balloonless catheter and a drip-infusion system to perform the retrograde study efficiently and comfortably for both the patient and the operator [6-8].

A few studies describe the potential diagnosis of CT voiding cystourethrography (CT-VCUG) to assess the bladder and posterior urethra after intravenous administration of iodized contrast $[9,10]$. However, the CT retrograde urethrography (CT-RUG) technique to evaluate anomalies of the male urethra has barely been reported [11]. The aim of the present study is to describe and evaluate a new technique for performing CT-RUG using the clamp method for urethrography in patients with urethral anomalies.

\section{Materials and Methods}

\subsection{Patients}

This prospective study was conducted between January 2017 and February 2020 in the Virgen de la Arrixaca University Hospital (Murcia, Spain). The study included consecutive males with urethral anomalies detected via urethrography and/or sonourethrography who received CT-RUG. Given the potential harm caused by radiation, subjects who had future reproductive plans were excluded. A time interval of more than a week between the urethrography and/or sonography and the CT, or any urethral manipulation (catheterization, dilation, cystoscopy, etc.) performed between the two studies, was also a criterion for exclusion. Prior to examination, all the subjects were informed of the entire scanning procedure, the significance of the study and the risks associated with exposure to X rays; they confirmed they had no iodine allergies and provided written informed consent. The study was approved by the Hospital Ethics Committee.

\subsection{Procedures}

\subsubsection{Clamp Method}

All the procedures were performed with the clamp method as described previously [6-8] using four elements: (1) a 6Fr pre-lubricated female urethral catheter (SpeediCath; Coloplast, Humlebæk, Denmark); (2) a B-Ring device (DVIMAG, Dispositivos Médicos de Imagen S.L., Albatera, Spain); (3) a catheter-infusion system connector (Vygon, Ecouen, France); and (4) an infusion system connected to a bottle of contrast positioned $2 \mathrm{~m}$ above floor level. The clamp method is performed in three steps:

1. Preparation of the material: the foreskin, if present, is retracted to permit sterile preparation of the glans penis with povidone-iodine and $2 \mathrm{~mL}$ of lubricating gel is introduced into the meatus. The catheter is connected to the infusion system and fastened in the catheter clamp on the B-Ring, and the catheter and infusion system are purged.

2. Compression: the catheter is inserted through the urethral meatus by lowering the B-Ring until the padded ring is level with the balanoprepucial sulcus and the band is pulled tight. The retrograde study is then conducted.

3. Removal: the band is cut with scissors, which releases the ring compression and enables the device and catheter to be removed easily.

\subsubsection{Urethrography}

The studies were conducted by a nurse with approximately 7 years' experience in the clamp method for urethrography using a fluoroscopy machine (Luminos Fusion, Siemens Healthcare, Erlangen, Germany); the parameters were as follows: $60-90 \mathrm{kV}, 20-80 \mathrm{mAs}$ and $150 \mathrm{~cm}$ of focus object distance. The infusion system was connected to a $250 \mathrm{~mL}$ bottle of iodinated contrast medium with a concentration of $165 \mathrm{mg}$ of Iodine/mL (Plenigraf, Juste Farma, Madrid, Spain). The patient was positioned obliquely at $45^{\circ}$ to the right, with the right leg resting on the table and hip and knee slightly flexed and the left leg in abduction with the foot resting on the table following flexion of the hip and knee. After compression with the B-Ring the penis was clamped and the device fastened with a strip of 
surgical tape to the skin of the right thigh, leaving the penis extended to avoid loops in the anterior urethra on RUG; the X-ray tube was angled $10^{\circ}$ cranially to avoid superposition of the bulbar urethra and the tuberosity of the ischium. Once the RUG was considered satisfactory, the patient was instructed to lie comfortably in the supine position while the strip of tape was removed to facilitate relaxation of the external sphincter and rapid filling of the bladder for VCUG.

\subsubsection{Retrograde Sonourethrography (RSUG)}

The studies were performed by a radiologist (J.B.M.) with 12 years' experience in RSUG using the clamp method. An ACUSON S2000 ultrasound system (Siemens Healthcare, Erlangen, Germany) was used together with a linear probe (range, 7-12 MHz). The infusion system was prepared by insertion of the connector into a 500-mL bottle of serum. The patient was placed in the Fowler semi-upright sitting position on a bed beside the ultrasound machine with his legs in the frog-leg position (hips and knees flexed). The penis was laid on the midline of the hypogastric region and immobilized by placing the middle of a 20-cm strip of surgical tape against the device and sticking the overlapping edges transversally over the skin of the two paraumbilical regions. The transducer was then placed lengthways on the ventral region of the penis and the infusion system turned on to visualize distension of the penile urethra; the transducer was then moved trans-scrotally and transperineally to visualize the bulbar urethra.

\subsubsection{Computed-Tomography Retrograde Urethrography (CT-RUG)}

The clamp method was performed in the CT room by the same nurse that performed the urethrography, with the extended penis positioned in the infraumbilical region and fastened with a strip of surgical tape in the same way as described for the RSUG but with the patient in the supine position and legs outstretched (Figure 1). A pelvic topogram was obtained in order to select the field of view (from the tip of the iliac crests to the lowest part of the scrotum). A 64 detector CT scanner was used (OPTIMA CT660, GE Healthcare, Chicago, Illinois, United States), with the following scan parameters: 0.6-s gantry rotation speed, high-quality scanning mode (64 detector array, $512 \times 512$ matrix, and a modulated $\mathrm{kV}$ of 120 and mAs interval of 60-550), and a $1.25 \mathrm{~mm}$ slice thickness (later reconstructed to a lower width of $0.625 \mathrm{~mm}$ ) at intervals of $0.625 \mathrm{~mm}$. The total scanning time, which depends on the length (total scan length of $22 \mathrm{~cm}$ ), was approximately $4 \mathrm{~s}$. The data were acquired craniocaudally and resulted in some 300 images per patient. One hundred millilitres of serum was removed from a $500 \mathrm{~mL}$ bottle and $50 \mathrm{~mL}$ of $370 \mathrm{mg} / \mathrm{mL}$ iodinated contrast (Ioprimide, Ultravist, Bayer, Leverkusen, Germany) was injected in its place to leave a bottle of $450 \mathrm{~mL}$ with a final concentration of some $40 \mathrm{mg}$ Ioprimide $/ \mathrm{mL}$, which was then connected to the infusion system. After performing the scanogram and collimating the field of study, we opened the infusion system, introduced about $50 \mathrm{~mL}$ of contrast and proceeded with the study. Cases with images of calcium or metal (urethral stent) detected either in previous studies or on the scanogram received a single sweep before the retrograde study. The cases that failed to achieve an adequate filling of urethrovesical contrast were given a second sweep after infusion of some $100 \mathrm{~mL}$ of contrast. 


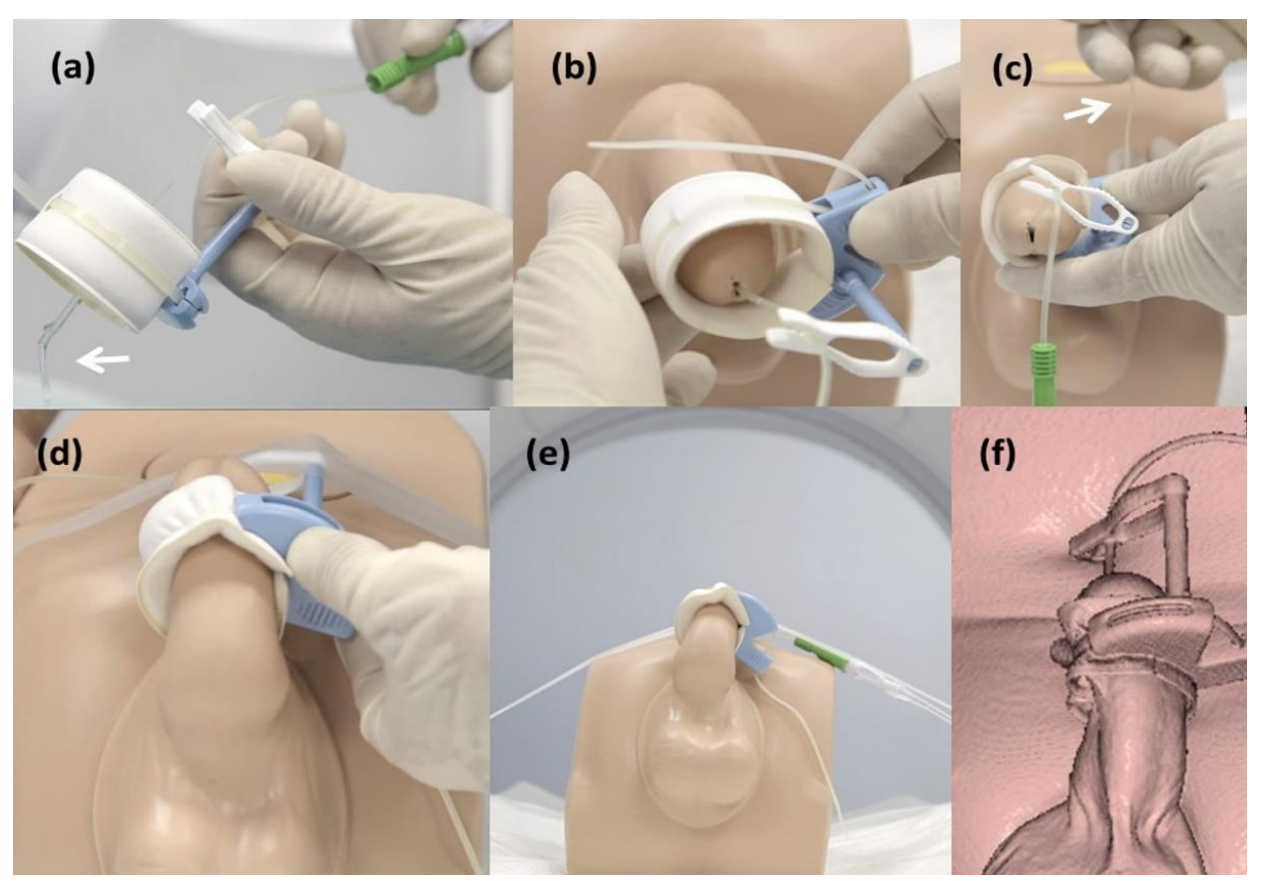

Figure 1. Description of the clamp method for Computed-Tomography (CT)-retrograde urethrography: (a) purging of the infusion system and catheter (arrow); (b) insertion of the catheter (held by the clamp) in the urethral meatus; (c) external compression when the band is pulled tight (arrow); (d) immobilisation of the penis in the infraumbilical region; (e) position prior to CT; (f) 3D CT image of one of the patients.

The transverse thin sections were transferred to a workstation (SyngoVia, Siemens Healthcare ) with manufacturer-provided software that allows the generation of 2D maximum intensity projection, 2D multiplanar reconstruction, 3D shaded-surface display, and $3 \mathrm{D}$ volume-rendered technique

\subsection{Data Collection}

All the data were collected by a single radiologist (A.N.B.). Clinical histories were collected to register the cause of the urethral anomalies.

The location and measurements of anterior urethral strictures were recorded on urethrography, RSUG and CT-RUG. A radiologist with 9 years' experience (F.G.A.) interpreted the urethrography study and a radiologist with 11 years' experience (A.P.S.) the CT-RUG, whereas the radiologist who performed the RSUG (J.B.M.) interpreted only that technique; the 3 radiologists were blind to the data and measurements of the other techniques. Patients with periurethral fistulas had location and measurements recorded only by urethrography and CT-RUG, as RSUG was not performed (a limitation for detecting filling of the fistula with anechoic serum); this was also the case for patients with an intraurethral stent (limitation of ultrasound to penetrate the metal). We also recorded fluoroscopy time and dose-area product [DAP: $\mu \mathrm{Gy} \cdot \mathrm{m}^{2}$ ] in the urethrography.

The following data were recorded for the CT-RUG: duration of the study (minutes between the patient being placed on the CT table and getting up again); the amount of contrast infused (ml); number of $\mathrm{CT}$ sweeps required for diagnosis, the dose of radiation (volume CT dose index (CTDIvol: mGy) and Dose Length Product (DLP: mGy.cm)); and level of pain with the clamp method, which was recorded on a visual analog scale (VAS) from 0 to 10 .

\subsection{Statistical Analysis}

The statistical analysis was performed using the software package SPSS Statistics for Windows (version 24.0, IBM, Armonk, NY, USA). A descriptive analysis of all the variables was conducted by determining the frequency distribution and the characteristic 
parameters of the quantitative variables (mean, standard deviation, maximum and minimum) were calculated. To compare the qualitative variables we used the Wilcoxon test and the Spearman's Rho test for quantitative variables. A $p$ value of $<0.05$ was considered statistically significant.

\section{Results}

Because of the risk of gonadal irradiation, we excluded 9 patients with reproductive plans in whom the diagnosis of the urethral anomaly was evaluated by urethrography and/or urethrosonography and another 3 patients who received urethral manipulation (urethrocystoscopy) between the urethrography/sonography and the CT. The study included 22 men with a mean age of $64 \pm 6.9$ (SD) years (range, 51-77 years) and CT-RUG with the clamp method was performed successfully in all of them. The patients were distributed into 3 groups according to their urethral pathology: anterior urethral stenosis $(n=11)$, periurethral fistula $(n=8)$ and those with a urethral stent $(n=3)$. RSUG was only done in the 11 cases with anterior urethral strictures, whereas CT-RUG and RUG were performed in all cases. VCUG was not done in 5 cases with periurethral fistulas (as RUG was enough to detect them) and VCUG revealed 3 cases with urethromeatal alterations: hypospadias $(n=1)$ and meatal strictures $(n=2)$.

Of the 11 cases with anterior urethral stenosis, most were located in the bulbar urethra $(n=7)$ and the rest in the penile or penobulbar urethra $(n=4)$. All the cases had previous urethral manipulation: radical retropubic prostatectomy $(n=2)$, urethroplasty $(n=2)$, transurethral resection of the prostate (TURP) $(n=2)$, internal urethrotomy $(n=2)$, urethrocystoscopy $(n=2)$ and urinary catheterisation $(n=1)$. In the 2 cases with urethroplasty the focal strictures between the tortuous lumen of the plasty were only revealed by CT-RUG (Figure 2). In the remaining 9 cases the mean length of the stricture was $2 \pm 0.7$ (SD) cm (range, 1.1-3.1 $\mathrm{cm}$ ) on urethrography, $2.8 \pm 0.9$ (SD) $\mathrm{cm}$ (range, 1.8-4.2 cm) on RSUG and $2.9 \pm 0.9(\mathrm{SD}) \mathrm{cm}$ (range, 1.9-4.3 cm) on CT-RUG. An underestimation of the stricture length was found on RUG when compared to RSUG and CT-RUG ( $p=0.008)$ (Figures 3 and 4).

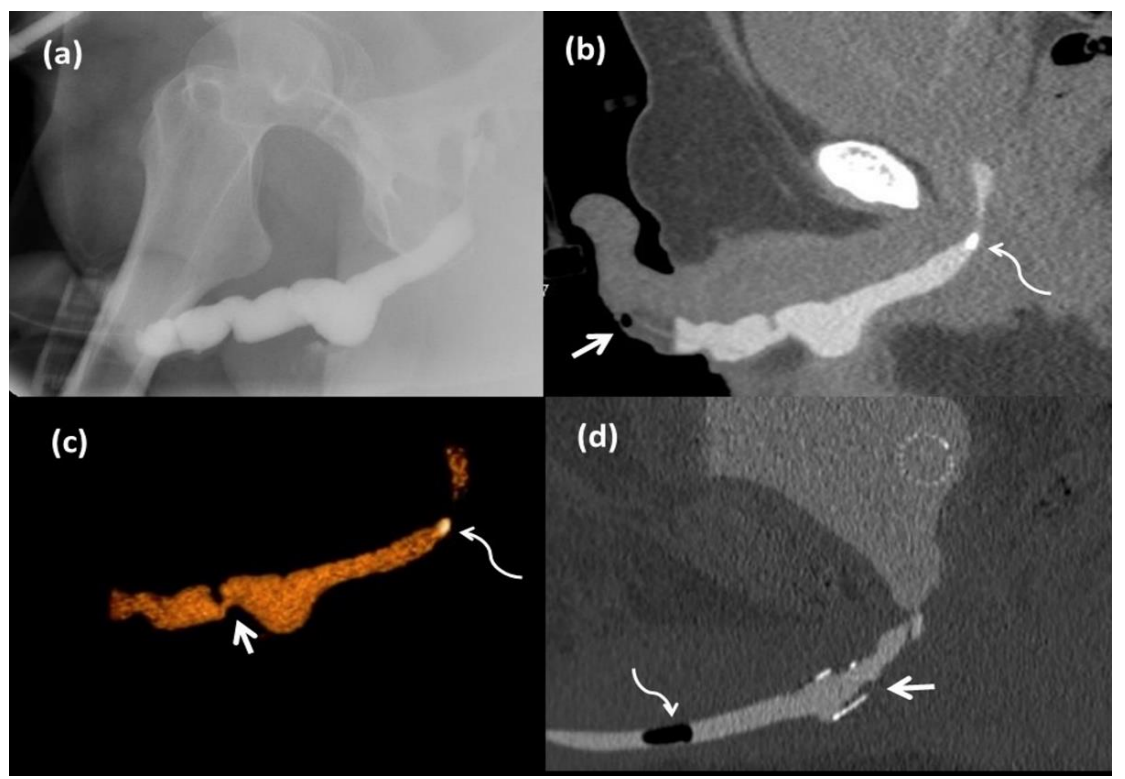

Figure 2. Urethral anomalies detected only by CT-retrograde urethrography: (a) retrograde urethrography (RUG) of patient with hypospadias and tortuous urethroplasty in the penile urethra, but no evident stenosis; (b) CT-RUG of the same patient showing bubble in hypospadic orifice (arrow), focal stenosis in urethroplasty and urethral lithiasis in bulbar cone (curved arrow); (c) 3D CT-RUG image of the same patient, showing focal stenosis in the urethroplasty (arrow) and urethral lithiasis (curved arrow), not visualized on RUG; (d) CT-RUG of the patient with bulbar urethral stent showing intraurethral bubble due to purging error (curved arrow) and discrete intra-stent spongiofibrosis (arrow) not visualized on RUG. 


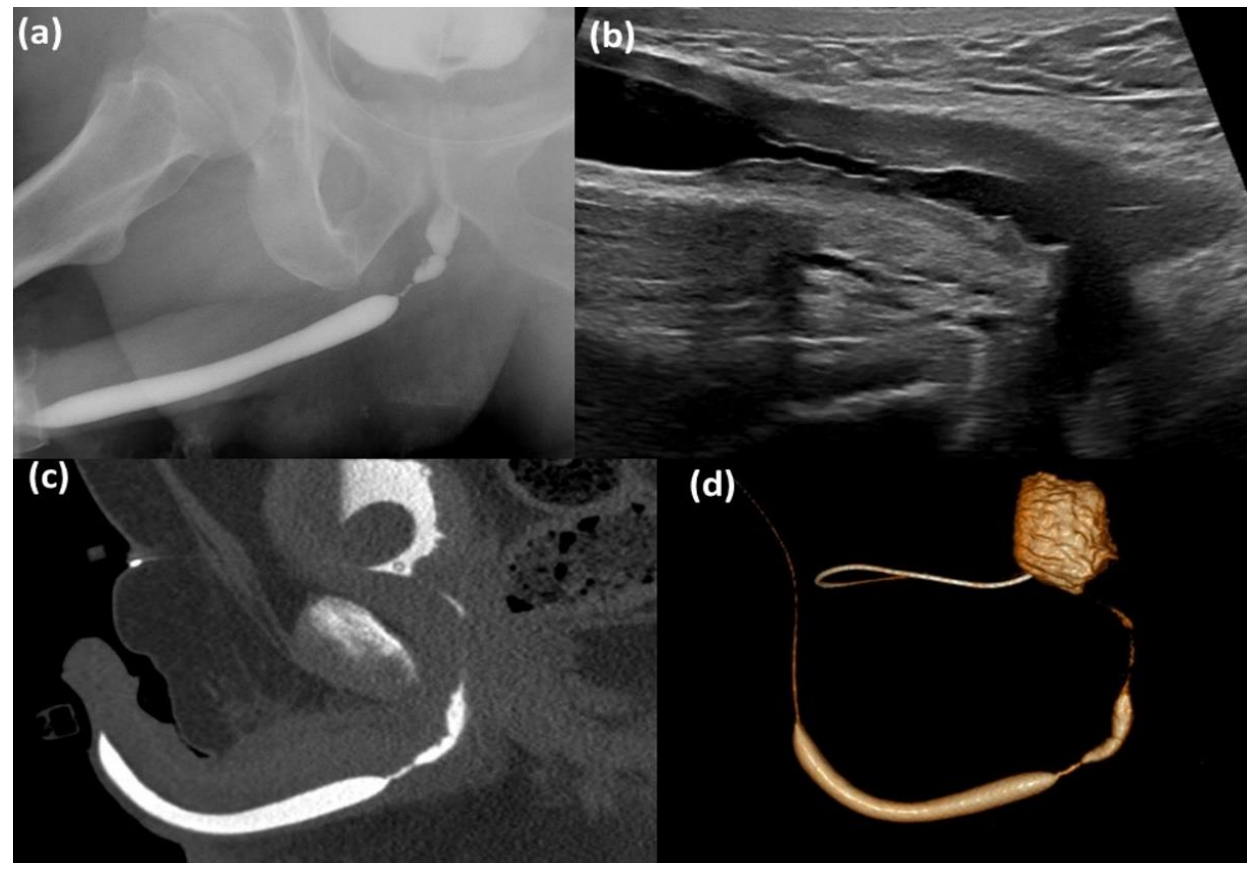

Figure 3. Patient with bulbar stenosis of a length underestimated by urethrography: (a) RUG showing $1.7 \mathrm{~cm}$ stenotic bulbar segment with a drastic reduction in the caliber of the urethral lumen; (b) retrograde sonourethrography (RSUG) showing the stenotic bulbar segment and surrounding spongiofibrosis, measuring $3.1 \mathrm{~cm}$ in total; (c) CT-RUG showing stenotic bulbar segment measuring $3.2 \mathrm{~cm}$ in length (measurement similar to RSUG and greater than RUG). (d): 3D CT-RUG image.

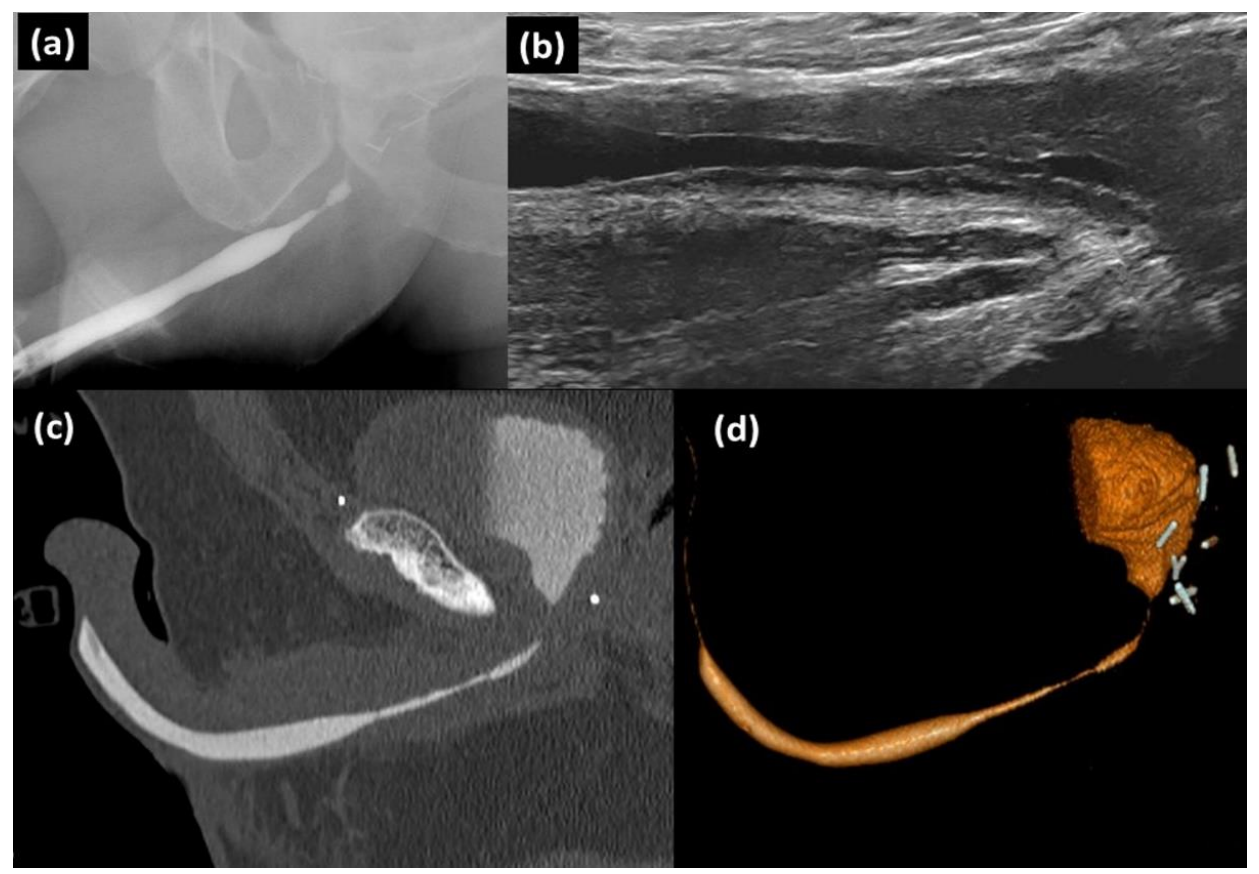

Figure 4. Patient with bulbar stenosis of a length underestimated by urethrography: (a) RUG showing $2.8 \mathrm{~cm}$ stenotic bulbar segment; (b) RSUG in panoramic mode (extended FOV) to visualise all of the bulbar stenosis and surrounding spongiofibrosis, measuring $4.2 \mathrm{~cm}$ in length; (c) CT-RUG showing $4.3 \mathrm{~cm}$ stenotic bulbar segment. (d): 3D CT-RUG image. 
The eight cases with periurethral fistulas had previous surgery: radical retropubic prostatectomy $(n=3)$, colorectal resection $(n=3)$, TURP $(n=1)$ and internal urethrotomy $(n=1)$. The fistula in most cases communicated the posterior urethra $(n=3)$ or bladder $(n=3)$ with the colorectal lumen; the case with internal post-urethrotomy showed fistulous tracts towards periurethral soft tissues and a further case had a urethrovesical fistula. CT-RUG proved more capable than urethrography of visualizing the fistulous tract: urethrography did not visualize the fistula in 3 of the 8 cases and it underestimated the length of the fistula in the other $5(1 \pm 0.5(\mathrm{SD}) \mathrm{cm}$ (range, $0.2-1.4 \mathrm{~cm})$ ) compared to CT-RUG $(1.4 \pm 0.6(\mathrm{SD}) \mathrm{cm}$ (range, $0.4-1.8 \mathrm{~cm}))(p=0.042)$ (Figure 5).

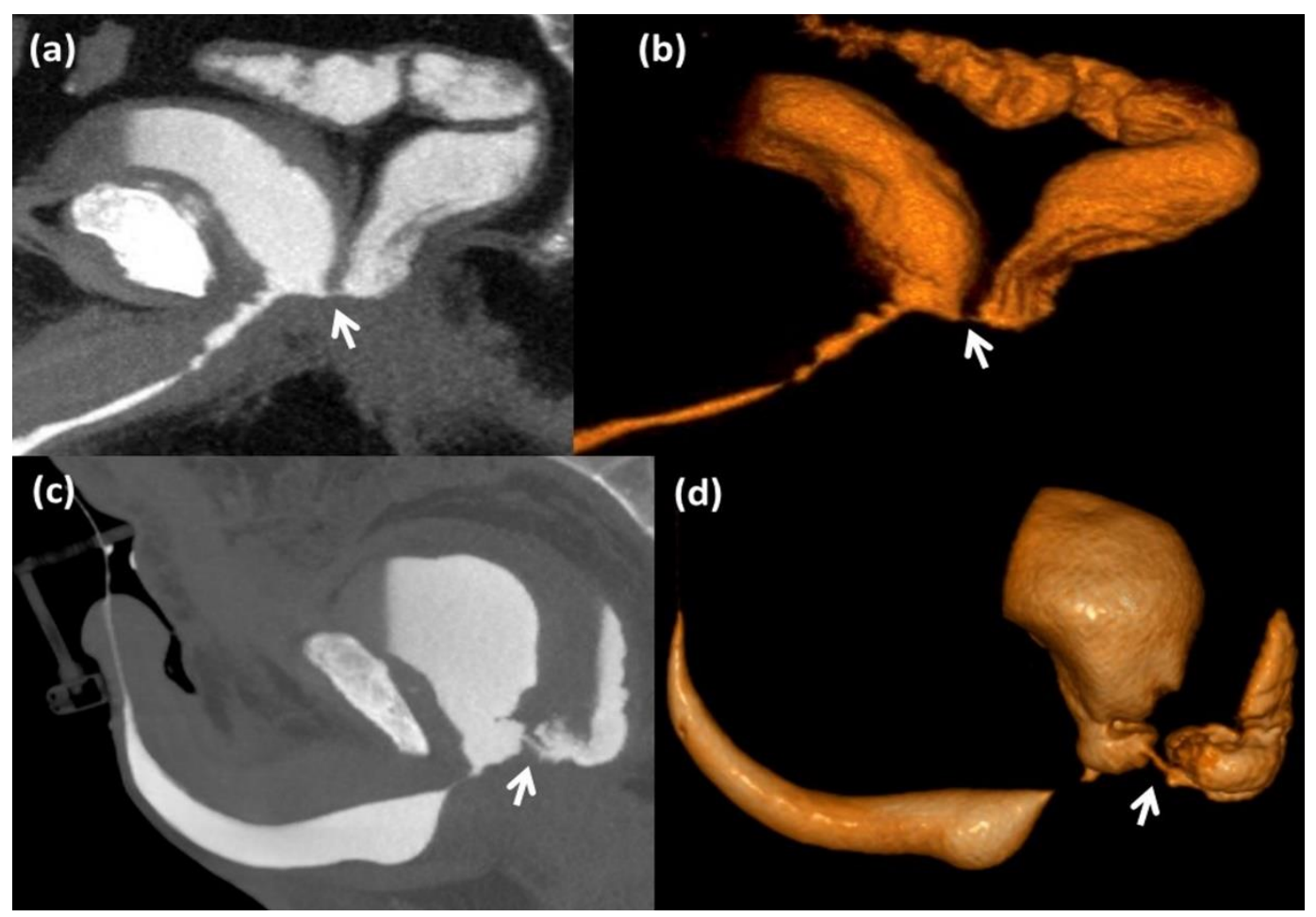

Figure 5. Fine-caliber periurethral fistulas visualized only by CT-retrograde urethrography: (a) CT-RUG showing smallcaliber vesicorectal fistula (arrow); (b) 3D CT-RUG image of the same case as A; (c) CT-RUG showing fistulous tract between neocavity of the posterior urethra and rectosigmoid (arrow); (d) 3D CT-RUG image of the same case as C.

In three cases fitted with a urethral stent, this was located more accurately with CT-RUG than with urethrography, as was the reduction in the intra-stent urethral caliber (Figure 2).

The mean duration of the CT-RUG with the clamp method was $6.1 \pm 0.8$ (SD) $\mathrm{min}$ (range, 4.7-7.7 $\mathrm{min}$ ) and the mean amount of contrast used was $95 \pm 21.9$ (SD) $\mathrm{ml}$ (range, $60-150 \mathrm{~mL}$ ). Of the 21 patients, $80.9 \%$ of the patients $(17 / 21)$ reported no pain (VAS: 0$)$ with the clamp method, three cases very mild pain (VAS: $0.5,0.8$ and 0.9 ) and one case mild pain (VAS: 1.2$)$. The patients with a urethral stent $(n=3)$ received 2 CT sweeps (one simple and one with contrast) and two cases with a periurethral fistula and another with urethral stenosis were also given two sweeps, as insufficient contrast was observed on the first sweep, such that the mean CTD $_{\text {Ivol }}$ in these six cases was $24.8 \mathrm{mGy}$ and the mean DLP was $588.5 \mathrm{mGy} . \mathrm{cm}$, with an effective dose of $4.96 \mathrm{mSv}$. The remaining 16 cases with anterior urethral strictures $(n=10)$ and periurethral fistulas $(n=6)$, who only had one CT sweep, received a mean $\mathrm{CTD}_{\mathrm{Ivol}}$ of $15.1 \mathrm{mGy}$ and a mean DLP of $284.4 \mathrm{mGy} . \mathrm{cm}$, with an effective dose of $3.456 \mathrm{mSv}$. The urethrography showed a mean fluoroscopy time of $5.9 \pm 1.1$ (SD) min (range, 3.5-6.8 $\mathrm{min}$ ) and a DAP of 3335,46 $\mu \mathrm{Gy} . \mathrm{m} 2$, with an effective dose of $0.67 \mathrm{mSv}$. 


\section{Discussion}

The introduction of contrast in RUG was first done using clamp devices coupled to a syringe (Knutsson clamp, Brodney clamp). These were replaced by the McCallum technique using a Foley catheter, but the drawbacks of this method are that it can cause pain on inflation of the balloon and it is not useful in cases with urethromeatal alterations, nor can anesthetic gel be used or contrast introduced very accurately (risk of balloon leakage) $[2,6]$. The technical difficulty with performing CT-RUG with a Foley catheter may be the cause. However, there are only 2 studies in which CT-RUG and CT-VCUG were done simultaneously to evaluate anomalies of the posterior urethra $[4,11]$. The first, by Kassaby et al. [4], evaluated urethral distraction defects after blunt pelvic trauma in 21 males; the bladder was filled through a suprapubic catheter (with which the patient was fitted) to achieve a full bladder sensation, after which they performed retrograde infusion of contrast (technique not specified) and combined retrograde and voiding CT. In the second study, Zhang et al. [11] evaluated posterior urethral strictures in 21 males; the bladder was filled with contrast via an infusion system connected to the suprapubic catheter (with which the patient was fitted) and the anterior urethra using a Foley catheter (after inflating the balloon with 2-3 mL of serum in the fossa navicularis), after which they performed the combined retrograde and voiding $\mathrm{CT}(3 \mathrm{~s}$ after the patient started to void). To our knowledge, the present study is the first to evaluate the utility of CT-RUG for stenosis of the anterior urethra (bulbar or penile). It reports an accuracy similar to RSUG and greater than RUG for measuring the length of strictures. In the 2 cases with urethroplasty the $\mathrm{CT}$ was also better than the other techniques for visualizing stenosis in the tortuous track of the plasty. The advantage of CT over urethrography is that it can evaluate periurethral structures and locate stenosis with greater anatomical accuracy. The advantage over RSUG is that CT has a complete field of view (FOV), whereas measuring stenotic segments longer than $3 \mathrm{~cm}$ means resorting to a panoramic ultrasound image, which may distort the measurement as it is particularly operator-dependent (Figure 4).

CT-RUG was performed in two studies in male Beagle dogs $[12,13]$. In the first both CT-RUG (using an 8Fr Foley catheter) and CT-VCUG were done in the dogs $(n=18)$ following urethroplasty of the anterior urethra and it was seen that CT is more accurate than urethrography for anatomical location and evaluation of the anomalies [12]. In the second study $(n=5)$ CT-RUG was performed using a $6 \mathrm{Fr}$ Foley catheter and a $200 \mathrm{~mL}$ syringe connected to a power injector, which made manual injection in the CT room unnecessary, and 3D anatomical images were produced of the male urethra in healthy dogs [13]. In the present study, CT-RUG was done successfully in all the cases; there was no need for manipulation of syringes or the presence of the operator in the room as drip infusion was used for the introduction of contrast. The amount of pain recorded by the patients for catheterization and external compression of the B-Ring was none or very mild. Although the clamp method can be done in cases with urethromeatal alterations (stenosis, hypospadias, meatotomy), when the balloonless fine probe is introduced through the anomaly the B-Ring exerts external compression just behind the corona of the glans penis, which is why the $2-4 \mathrm{~cm}$ of the distal urethra (inside the glans) is not visualized in the retrograde study, where the distended penile urethra is observed beyond the balanoprepucial sulcus. In the present study, the urethromeatal anomalies were visualized by VCUG, and during micturition contrast was seen to flow through the hypospadic orifice or the meatal stenosis (with prestenotic dilatation). Future studies are needed with CT-VCUG to analyze its capacity to visualize urethromeatal anomalies.

Some studies report the usefulness of CT-VCUG for evaluating the bladder and posterior urethra [5,9,10]. Chou et al. [9] in a study of 13 males with traumatic antecedents reported the diagnostic potential of 3D CT-VCUG after intravenous (i.v.) administration of iodinated contrast. Su et al. [10] described 3D and 4D CT-VCUG after i.v. the contrast in 70 healthy subjects (43 males and 27 females) using 640-slice dynamic volume CT. Two studies using CT-VCUG [5,9] took a mean time of 8.8 and 9 min (range, 4-20 min) and another performing combined retrograde and voiding CT [11] took a mean time of $10 \mathrm{~min}$ 
(range, 6-20 min). The mean time for CT-RUG in our study was $6.1 \mathrm{~min}$ (range, 4.7-7.7 $\mathrm{min}$ ) and it must be remembered that a lot of this time was spent by the nurse performing the clamp method because the $\mathrm{CT}$ technique and cystourethral filling with contrast were quick. The method described in the present study may enable future CT-VCUG studies to be done with retrograde bladder filling, as the bottle of contrast is positioned $2 \mathrm{~m}$ from the floor, which means there is sufficient pressure for rapid bladder filling and avoids i.v. administration of contrast to performing CT-VCUG. As for the dose of radiation received by the patient, which is one of the main drawbacks of $\mathrm{CT}$, the mean was 4.96 $\mathrm{mSv}$ in our patients with 2 sweeps and $3.456 \mathrm{mSv}$ in those with one sweep, whereas the mean effective dose in the CT-VCUG study [10] was $6.585 \mathrm{mSv}$, which is in line with the International Commission on Radiological Protection requirements. One of the advantages of urethrography over CT-RUG is that it is a dynamic study that allows filling of the urethral alterations to be visualized in real-time. Moreover, the effective mean dose obtained on urethrography was $0.67 \mathrm{mSv}$, which shows significantly greater irradiation of CT-RUG than with fluoroscopy. This is why CT is not recommended in males of childbearing age and why nine cases with reproductive plans were excluded from the study (because of the risk of gonadal irradiation); it is also why the study patients were aged over 50 years. In our opinion, further studies are necessary to adapt the CT protocol to lower doses of radiation.

There is little published on periurethral fistulas, in which the relevance of CT-UG for diagnosis is reflected $[5,14,15]$. Xiang Guo Lv et al. [5] performed CT-VCUG in 80 patients (58 males and 22 females) after bladder filling by suprapubic catheter infusion (or retrograde); of the 58 males with posterior urethral strictures with fistulas a higher number was visualized by CT $(n=51)$ than by urethrography $(n=34)$, such that the fistula detection rate of CT-VCUG was higher than that of urethrography $(87.9 \%$ vs. $58.6 \% ; p=0.015)$. In our study, CT-RUG enabled the fistulous tracts to be visualized in all the cases $(n=8)$, whereas only five cases were visualized by urethrography. Although CT-VCUG was not done in our study, retrograde filling of the urethra and bladder with a mean of $90 \mathrm{~mL}$ of contrast allowed us to visualize fistulas of the bladder and posterior urethra, which shows the diagnostic potential of this technique to detect periurethral fistulas without the patient having to micturate.

One of the limitations of the study is the low number of cases $(n=22)$, though it must be remembered that 12 cases were excluded and that it was difficult to include more cases in a threee-year period because it was not an extended technique. Another limitation is that no surgical correlation was made in all the cases, because except for those with urethral fistulas and some with stenosis of the anterior urethra the rest had no posterior urological intervention (except dilations). A further limitation is that cases with stenosis of the posterior urethra were not included, because as occurs with VCUG we would have had to perform CT-VCUG in order to visualize adequate distension and evaluate posterior urethral strictures (due to the external sphincter stopping the passage of contrast during micturition). Lastly, we should point out that CT-RUG was not used in our study to measure the thickness of the spongiofibrosis surrounding the anterior urethral stenosis; in our opinion, RSUG is the technique of choice, as ultrasonography enables us to differentiate between the echogenicity of the spongiofibrosis and the periurethral tissues, whereas the scar tissue surrounding the stenosis appears isodense on CT (water density) in respect of the unaffected corpus spongiosum and the cavernous bodies, which does not allow the spongiofibrosis to be measured accurately.

El Kassaby et al. [4] first described the CT-UG technique in 2003 and after comparing it to urethrography concluded that CT-UG was more informative in several aspects: the location and the length of the posterior urethral distraction defect, the direction of alignment or misalignment, the bone anatomy (ectopic fragments, callus) and the presence of additional urinary pathology (fistulae, false passages and diverticulae). These advantages of CT-UG have been confirmed in later studies [5,9-15]; however, it is still not a widely used technique in clinical practice. 


\section{Conclusions}

The present study describes a new technique for performing CT-UG, which is convenient for both the patient (it causes no discomfort) and the operator (the bladder is filled retrograde by infusion), such that it may increase the indications and applications of CT-UG.

Author Contributions: Conceptualization, J.d.D.B.-M.; methodology, J.d.D.B.-M. and F.G.-A.; validation, A.P.-S., J.d.D.B.-S. and M.A.; statistical analysis, F.G.-A.; resources, J.d.D.B.-M., F.G.-A. and A.P.-S.; data curation, A.N.-B.; writing-original draft preparation, J.d.D.B.-M. and A.N.-B.; writing-review and editing, G.C.-L.d.C. and M.A.; visualization, J.d.D.B.-M. and A.P.-S.; supervision, J.d.D.B.-S. and M.A. All authors have read and agreed to the published version of the manuscript.

Funding: This research received no external funding.

Institutional Review Board Statement: The study did not require ethical approval because the diagnostic examination is systematically protocolized at this Center.

Informed Consent Statement: Informed consent was obtained from all subjects involved in the study.

Data Availability Statement: The data used in this study are found in the medical records of the patients examined. They require a specific authorization for their review.

Acknowledgments: The authors would like to thank Enrique Pardo Mateo, the nurse who performed the clamp method in the urethrography and CT urethrography studies.

Conflicts of Interest: The authors declare no conflict of interest.

\section{References}

1. Kim, B.; Kawashima, A.; LeRoy, A.J. Imaging of the male urethra. Semin. Ultrasound CT MR 2007, 28, 258-273. [CrossRef] [PubMed]

2. Maciejewski, C.; Rourke, K. Imaging of urethral stricture disease. Transl. Androl. Urol. 2015, 4, 2-9. [PubMed]

3. Theisen, K.M.; Kadowm, B.T.; Rusilko, P.J. Three dimensional imaging of urethral stricture disease and urethral pathology for operative planning. Curr. Urol. Rep. 2016, 17, 54. [CrossRef] [PubMed]

4. El-Kassaby, A.W.; Osman, T.; Abdel-Aal, A.; Sadek, M.; Nayef, N. Dynamic three-dimensional spiral computed tomographic cysto-urethrography: A nove technique for evaluating post-traumatic posterior urethral defects. BJU Int. 2003, 92, 993-996. [CrossRef] [PubMed]

5. Lv, X.G.; Peng, X.F.; Feng, C.; Xu, Y.M.; Shen, Y.L. The application of CT voiding urethrography in the evaluation of urethral stricture associated with fistula: A preliminary report. Int. Urol. Nephrol. 2016, 48, 1267-1273. [CrossRef] [PubMed]

6. Berná-Mestre, J.D.; Berná-Serna, J.D.; Aparicio-Mesón, M.; Canteras-Jordana, M. Urethrography in men: Conventional technique versus clamp method. Radiology 2009, 252, 240-246. [CrossRef] [PubMed]

7. Berná, J.D.; Berná, J.D., Jr.; Aparicio-Mesón, M. Urethrography in the male: The clamp method. Acta Radiol. $2009,50,233-237$.

8. Berná-Mestre, J.D.; Balmaceda, T.; Martínez, D.; Escudero, J.F.; Martínez, G.; García, J.A.; Canteras-Jordana, M.; Berná-Serna, J.D. Optimisation of sonourethrography: The clamp method. Eur. Radiol. 2018, 28, 1961-1968. [CrossRef] [PubMed]

9. Chou, C.P.; Huang, J.S.; Wu, M.T.; Pan, H.B.; Huang, F.D.; Yu, C.C.; Yang, C.F. CT Voiding Urethrography and Virtual Urethroscopy: Preliminary Study with 16-MDCT. AJR Am. J. Roentgenol. 2005, 184, 1882-1888. [CrossRef] [PubMed]

10. Su, Y.; Fang, K.; Mao, C.; Xiang, S.; Wang, J.; Li, Y. 640-slice DVCT multi-dimensionally and dynamically presents changes in bladder volume and urine flow rate. Exp. Ther. Med. 2018, 15, 2557-2562. [CrossRef] [PubMed]

11. Zhang, X.M.; Hu, W.L.; He, H.X.; Lv, J.; Nie, H.B.; Yao, H.Q.; Yang, H.; Song, B.; Peng, G.M.; Liu, H.L. Diagnosis of male posterior urethral stricture: Comparison of 64-MDCT urethrography vs. standard urethrography. Abdom. Imaging 2011, 36, 771-775. [CrossRef] [PubMed]

12. Orabi, H.; Aboushwareb, T.; Tan, J.; Yoo, J.J.; Atala, A. Can computed tomography-assisted virtual endoscopy be an innovative tool for detecting urethral tissue pathologies? Urology 2014, 83, 930-936. [CrossRef] [PubMed]

13. Kang, K.; Kim, K.; Oh, D.; Choi, J.; Choi, M.; Yoon, J. Retrograde CT urethrography using a power injector quantitatively reveals effects of bladder distension on urethral size in healthy male Beagle dogs. Vet. Radiol. Ultrasound 2020, 61, 302-311. [CrossRef]

14. Yu, N.C.; Raman, S.S.; Patel, M.; Barbaric, Z. Fistulas of the genitourinary tract: A radiologic review. Radiographics 2004, 24, 1331-1352. [CrossRef] [PubMed]

15. Parlak, S.; Okay, A.E. Urethroscrotal Fistula: A Rare Cause of Scrotal Swelling. Pol. J. Radiol. 2016, 81, 438-440. [CrossRef] 